\title{
Orientation de la charnière d'un kink-band
}

\author{
Kink-band hinge orientation
}

\author{
P. HABIB \\ Laboratoire de Mécanique des Solides* \\ (Ecole Polytechnique - ENSMP - ENPC - Associé au CNRS)
}

Rev. Franç. Géotech. n 56, pp. 51-55 (juillet 1991)

\section{Résumé}

L'orientation de la charnière d'un kink-band est en relation avec I'angle de dilatance des feuillets lors d'un cisaillement parallèle à la foliation.

\footnotetext{
Abstract

Kink-band hinge orientation is related with the angle of dilatancy of a shear strain parallel to the foliation.
} 


\section{INTRODUCTION}

Un « kink-band " se produit lorsqu'un matériau très anisotrope, comme une roche schisteuse, est sollicité en compression dans le plan de la foliation (1) (3). La photographie de la figure 1 montre un kink-band bien développé dans un échantillon de schiste provenant du col de Seigne, entre la France et l'Italie, dans le massif du Mont-Blanc. L'épaisseur de la bande déformée en bayonnette est ici égale à l'épaisseur de l'empilement schisteux initial ; il en résulte que la charnière du kink est bissectrice de l'angle du kink si l'on admet que la longueur des feuillets dans la bande est égale à sa longueur initiale, et si le volume de la bande est resté égal au volume initial après la transformation ayant engendré le plissement, ce qui semble conforme à la réalité, la roche étant aussi compacte dans le pli que hors du pli. En général, les angles caractéristiques des "kink-bands " ne sont pas souvent aussi réguliers que dans le présent exemple et la charnière du kink est rarement exactement bissectrice de l'angle du kink (4). Du fait de l'enchevêtrement des kinks et de leur interaction tridimensionnelle, peut-être aussi

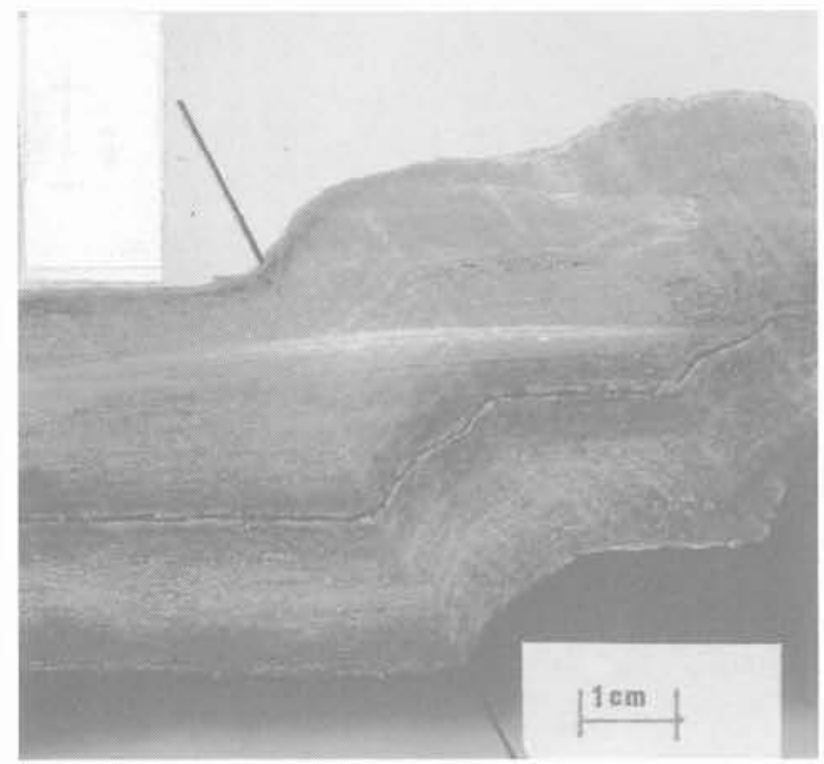

Fig. 1. - Photographie d'un kink-band bien développé. Fig. 1. - Well formed kink-band photography des déformations tectoniques ultérieures, la dispersion des angles mesurés est forte, peut-être de l'ordre de $\pm 5^{\circ}$. Les notations sont précisées sur la figure 2 ; $2 \alpha$ est l'angle du kink ; $\pi-2 \alpha$ est la déviation des feuillets: l'angle de la charnière du kink avec la normale à la foliation est $\frac{\pi}{2}-\alpha$. L'expérience montre que cet angle est toujours inférieur à $\frac{\pi}{4}$ ce qui indique que ce phénomène est sans rapport avec la formation d'un plan de glissement qui, lui, serait toujours plus incliné que $\frac{\pi}{4}$. Il s'agit en fait d'une instabilité analogue à un flambage local.

\section{MODÉLISATION}

Examinons l'instabilité du modèle mécanique suivant. Les feuillets du schiste dans le kink sont remplacés par un empilement de plaquettes articulées à leurs extrêmités, les points d'articulation étant alignés sur deux droites parallèles inclinées de l'angle $\frac{\pi}{2}-\alpha$ sur la normale aux feuillets (fig. 3).

Sous l'effet d'une contrainte dans le plan des feuillets, le système est instable d'un seul côté, vers le haut sur la figure 3 . Au début du mouvement, lorsque les

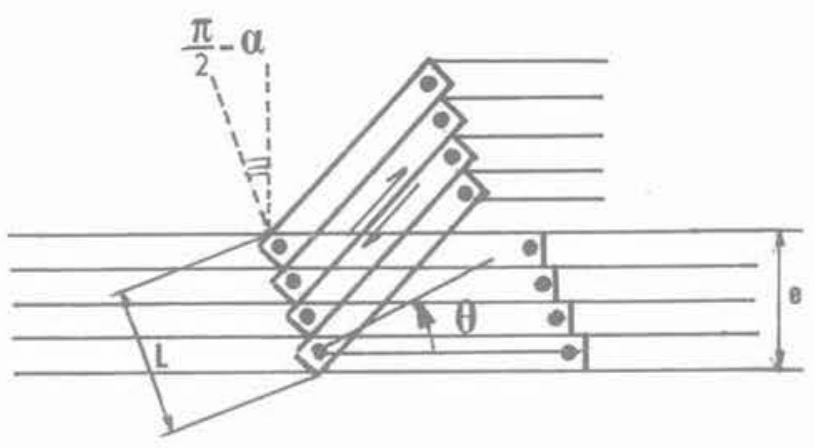

Fig. 3. - Modélisation.

Fig. 3. - Modelisation.
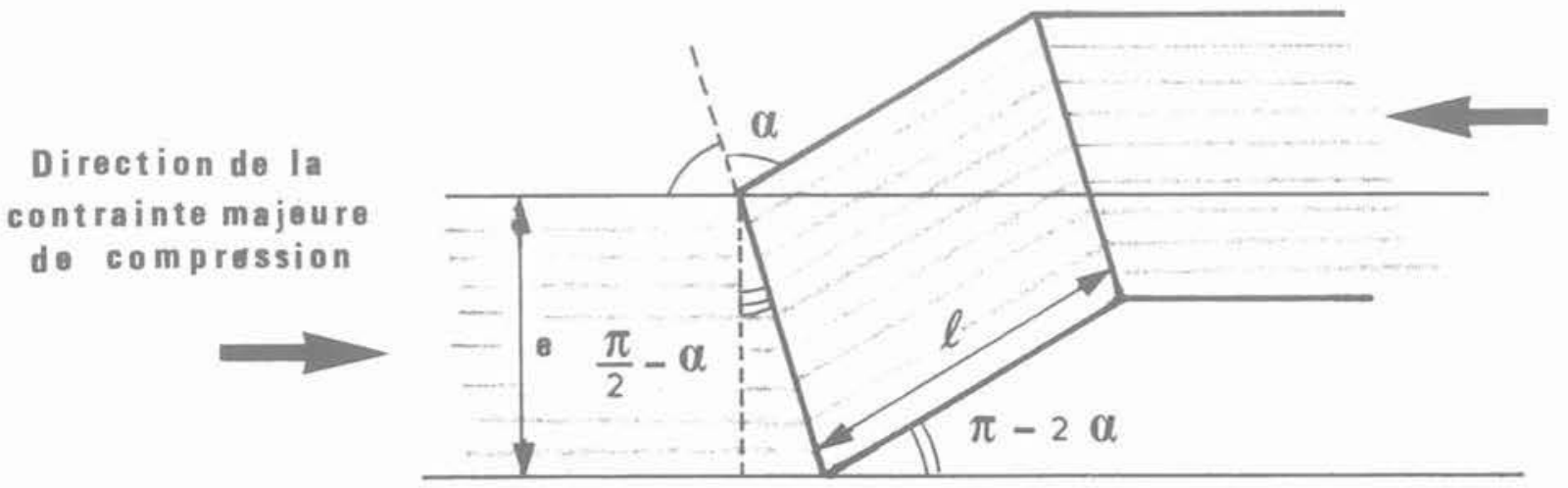

Fig. 2. - Notations.

Fig. 2. - Notations. 
plaquettes tournent d'un angle $\theta$, elles s'écartent les unes des autres; l'épaisseur de la bande articulée passe par un maximum pour $\theta=\frac{\pi}{2}-\alpha$ puis revient à l'épaisseur initiale e lorsque $\theta=\pi-2 \alpha$. A ce moment, le mécanisme se bloque et ne peut aller plus loin. Il est facile de calculer la variation d'épaisseur en fonction de $\theta$.

La longueur L de la charnière est :

$$
\mathrm{L}=\frac{e}{\sin \alpha} .
$$

L'épaisseur $e(\theta)$ de l'empilement est :

$$
e(\theta)=e \frac{\sin (\alpha+\theta)}{\sin \alpha} .
$$

La déformation d'épaississement, c'est-à-dire la dilatation normale est :

$$
\begin{gathered}
\epsilon_{n}=\frac{e(\theta)-e}{e}=\frac{\sin (\alpha+\theta)}{\sin \alpha}-1 \\
\frac{d e(\theta)}{d \theta}=e \frac{\cos (\alpha+\theta)}{\sin \alpha} \\
\text { nul pour } \theta=\frac{\pi}{2}-\alpha .
\end{gathered}
$$

La plus grande valeur de $\frac{\mathrm{d} e(\theta)}{\mathrm{d} \theta}$ se produit pour :
$\theta=0$ :

$$
\begin{aligned}
& \frac{d e(\theta)}{d \theta}(\theta=0)=\frac{e}{\operatorname{tg} \alpha} \\
& =e \operatorname{tg}\left[\frac{\pi}{2}-\alpha\right]
\end{aligned}
$$

qui est identique évidemment, au signe près, à

$$
\frac{\mathrm{d} e(\theta)}{\mathrm{d} \theta}(\theta=\pi-2 \alpha) \text {. }
$$

La dilatation normale pour $\theta=0$ est donc une fonction croissante de $\frac{\pi}{2}-\alpha$ : au début de l'instabilité, le volume du modèle augmente et il augmente d'autant plus que l'angle $\frac{\pi}{2}-\alpha$ est grand. Si l'empilement de plaquettes est situé dans un champ de contraintes triaxiales, avec une contrainte non négligeable $\sigma_{3}$ perpendiculaire au plan des feuillets, le travail de la dilatation normale s'oppose à l'instabilité et cela d'autant plus que $\frac{\pi}{2}-\alpha$ est grand. Il en résulte que l'instabilité se produira préférentiellement pour l'angle de charnière $\frac{\pi}{2}-\alpha$ le plus petit pos-
sible.

Considérons maintenant le mouvement relatif de deux plaquettes (fig. 4). Une plaquette étant considérée

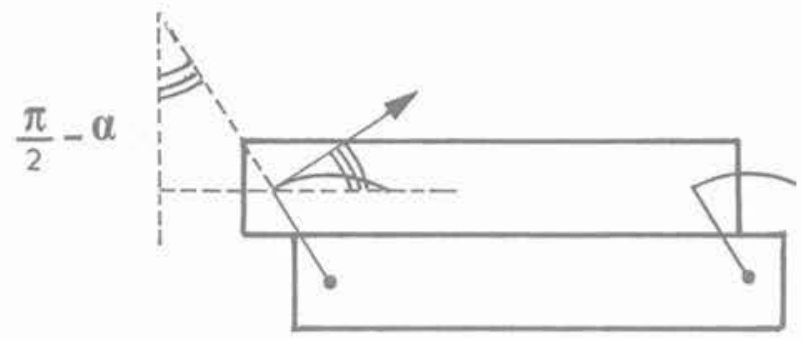

Fig. 4. - Mouvement relatif de deux plaquettes.

Fig. 4. - Relative movement of two small plates.

comme fixe, la voisine est animée d'un mouvement de translation circulaire et l'angle de la tangente au cercle avec le plan des feuillets au début du mouve-

ment est $\frac{\pi}{2}-\alpha$.

Mais les feuillets minéraux ne sont pas des plans parfaits et si on cherche à les faire glisser l'un sur l'autre au début du mouvement, il se produit une dilatance $\delta$ liée aux irrégularités des surfaces en contact. Si $\delta$ est plus grand que $\frac{\pi}{2}-\alpha$, le mouvement des plaquettes est bloqué et l'instabilité ne peut commencer : l'ensemble des plaquettes forme un monolithe. Ainsi, l'angle de la charnière avec la normale aux feuillets doit d'une part, être le plus petit possible et d'autre part, il ne peut pas être inférieur à l'angle de dila-

tance. On a donc $\frac{\pi}{2}-\alpha=\delta$. Sur la figure 1 , on lit $\frac{\pi}{2}-\alpha=24^{\circ}$.

\section{DISCUSSION}

La modélisation proposée est évidemment très simplifiée ; elle ne tient pas compte par exemple, de l'existence d'une cohésion entre feuillets, comme s'il s'agissait des pages d'un livre. Cependant, dans un champ de contraintes triaxiales, les actions de contact par frottement sont certainement prédominantes pour un matériau très anisotrope. Elle ne tient pas compte non plus que la contrainte principale majeure peut ne pas être rigoureusement parallèle à la foliation.

Mais le kink se développe-t-il d'une pièce entre deux charnières ou bien, comme c'est souvent admis (1) (2), s'élargit-il progressivement comme le font les bandes de Luders? Les expériences, en essai triaxial à température ambiante, sur des échantillons de gypse fer de lance chargés dans une direction parallèle aux plans de clivage ont semblé nous montrer que c'était plutôt le premier mécanisme qui se produit. Mais alors quelle serait la longueur des feuillets dans la bande? Il est évident que le modèle proposé est trop simple pour pouvoir répondre à cette importante question.

On peut cependant essayer d'approfondir ce point. Si $\sigma_{3}$ est la pression de confinement autour du matériau au moment de la formation du kink, $\left(\sigma_{1}-\sigma_{3}\right)$ est la contrainte déviatorique située par hypothèse dans le plan des feuillets. 
La force tangentielle $\phi$ exercée par ce déviateur sur la facette inclinée à $\frac{\pi}{2}-\alpha$ (c'est-à-dire la direction du kink-band) dans un empilement d'épaisseur e (fig. 2) est :

$$
\phi=\left(\sigma_{1}-\sigma_{3}\right) \text { e } \cos \alpha
$$

Le volume initial du matériau qui va former le kinkband (fig. 2) est $\ell$ e (pour une épaisseur unitaire). Après la rotation $\theta$ on calcule aisément que ce volume devient $V_{(\theta)}=$ le $\frac{\sin (\alpha+\beta)}{\sin \alpha}$ dont la dérivée est $V^{\prime}(\theta)=l e \frac{\cos (\alpha+\beta)}{\sin \alpha}$. La variation du volume $\Delta \mathrm{V}$ est donc égale à :

$$
\Delta \mathrm{V}: \mathrm{le} \frac{\cos \alpha \cos \theta-\sin \alpha \sin \theta}{\sin \alpha} \Delta \theta
$$

et pour $\theta$ petit il vient :

$$
\Delta V=l e(\operatorname{cotg} \alpha-\theta) \Delta \theta
$$

Le travail de la dilatance est $\Delta \mathrm{W}=\sigma_{3} \Delta \mathrm{V}=\sigma_{3}$ le ( $\operatorname{cotg} \alpha-\theta) \Delta \theta$ et ce travail est équivalent à celui d'une force tangentielle $\mathrm{F}$ appliquée sur la face inclinée du kink-band. Le travail de cette force lors du glissement $\Delta \theta$ serait F. $\ell \Delta \theta \cdot \sin \alpha$. Le travail de la dilatance est donc équivalent à celui d'une force d'opposition $\mathrm{F}=\frac{\sigma_{3} \text { e }(\operatorname{cotg} \alpha-\theta)}{\sin \alpha}$. On remarque que cette expression est indépendante de $\ell$.

Tant que la force $\phi$ engendrée par le déviateur est inférieure à $\mathrm{F}$ il ne se passe aucun mouvement, mais si $\sigma_{1}$ augmente et que $\phi$ dépasse $F$ d'une quantité $\Delta \phi$, il peut se produire une rupture en kink puisque F décroit avec $\theta$, et il $y$ a instabilité puisque le kink peut se produire vers la droite ou vers la gauche. Pour calculer $\theta$, on peut écrire l'équation d'équilibre:

$$
\begin{gathered}
\Delta \phi+\left[\left(\sigma_{1}-\sigma_{3}\right) e \cos \alpha-\sigma_{3} e \frac{\cos \alpha}{\sin ^{2} \alpha}\right] \\
{[\phi-F]=0} \\
+\frac{\sigma_{3} e \theta}{\sin \alpha}=\frac{\mathrm{Ge} \theta}{\sin \alpha}
\end{gathered}
$$

où $\mathrm{G}$ est le module de cisaillement de la roche.

D'où :

$$
\theta=\frac{\Delta \phi}{\frac{e}{\sin \alpha}\left(G-\sigma_{3}\right)}
$$

On voit que $\theta$ devient infini pour $\sigma_{3}=G$ ce qui signifie qu'il y a alors instabilité.
Il est évidemment extrêmement difficile d'évaluer G à long terme d'un ensemble feuilleté surtout si l'on tient compte qu'il existe une cohésion (à long terme elle aussi) entre les feuillets, peut-être d'ailleurs fonction de $\sigma_{3}$. En fait, il faut simplement interpréter cette relation en disant que pour que le kink se produise, il faut que la pression de confinement soit supérieure ou égale à une certaine valeur, fonction du matériau et dans ce cas, le kink peut se produire avec une longueur de feuillets indéterminée puisque $\ell$ ne figure pas dans l'équation d'équilibre. Le rapport entre l'épaisseur des feuillets élémentaires et leur longueur $\ell$ doit évidemment intervenir pour éviter que les feuillets ne flambent et se raccourcissent lorsqu'ils cessent d'être en contact avec leurs voisins au cours de la rotation $\theta$ et on ne peut pas imaginer des épaisseurs de kink-band très grandes. S'il y a un raccourcissement des feuillets, la direction du kink-band cesse d'être bissectrice de l'angle de kink $2 \alpha$, l'angle extérieur à la bande étant plus petit que l'angle dans la bande ce qui semble se vérifier statistiquement expérimentalement (4).

\section{CONCLUSIONS}

Avec les hypothèses adoptées, la modélisation proposée pour interpréter la formation d'un kink-band aboutit aux conclusions suivantes :

- la direction du kink-band est voisine de la bissectrice de l'angle $2 \alpha$ du kink. S'il y a raccourcissement (ou plissotement) des feuillets dans le kink l'angle extérieur à la bande est plus petit que l'angle extérieur à la bande :

- l'angle entre la direction de la bande et la normale aux feuillets de la schistosité est égal à l'angle de dilatance du glissement des feuillets les uns sur les autres ;

- l'épaisseur de la bande pliée n'est pas un paramètre du modèle. Elle peut donc être quelconque dans des limites raisonnables et on peut même imaginer des mécanismes de kink-bands en sifflet.

Le kink-band ne peut apparaître que si la pression de confinement autour du milieu feuilleté dépasse une certaine valeur.

- Enfin, si la contrainte $\sigma_{3}$ augmente fortement, les forces de contact entre feuillets rétablissent la continuité mécanique ; il est bien connu alors que l'anisotropie s'efface et que si la rupture se produit, elle se matérialise par une surface de glissement coupant les feuillets et non plus par des kink-bands. Les orientations sont alors tout à fait différentes.

\section{BIBLIOGRAPHIE}

(1) APARICO M., SIRIEYS P. (1978), Déformation naturelle des roches par kink-bands. Colloque Int. "Sciences de la terre et mesures ». Orléans 1977. Mémoires du BRGM, nº 91 (1978), pp. 187-198.

(2) DONATH F.A. (1968), Experimental study of kink-band development in Martinsburg slate in "Kink Bands and Brittle Deformation ». Géol. Surv. Pap. Can. $68-52$, pp. 255-288. 
(3) SIRIEYS P. (1985), Mécanismes de glissement plastique des roches à structure planaire. Coll. Int. du CNRS n 319 - "Comportement plastique des solides anisotropes ", pp. 475-488.
(4) STUBLEY M.P. (1990), The geometry and kinematics of a suite of conjugate kink bands, southeastern Australia. Journal of Structural Geology. Vol. 12, n० 8, 1990, pp. 1019-1031. 